\title{
MODELLING AND SIMULATION OF THE THREE-DIMENSIONAL HYDRODYAMIC PROBLEM
}

\author{
Matthieu TOURBIER \\ Laboratoire de Mécanique et Matériaux \\ Division Mécanique des Structures \\ Ecole Centrale de Nantes - 1, rue de La Noë \\ 44321 Nantes Cedex 3, France \\ matthieu.tourbier@ec-nantes.fr
}

\author{
Bundi DONGUY \\ Laboratoire de Mécanique et Matériaux \\ Division Mécanique des Structures \\ Ecole Centrale de Nantes - 1, rue de La Noë \\ 44321 Nantes Cedex 3, France \\ bundi.donquy@ec-nantes.fr
}

\author{
Bernard PESEUX \\ Laboratoire de Mécanique et Matériaux \\ Division Mécanique des Structures \\ Ecole Centrale de Nantes - 1, rue de La Noë \\ 44321 Nantes Cedex 3, France \\ bernard.peseux@ec-nantes.fr \\ Laurent GORNET \\ Laboratoire de Mécanique et Matériaux \\ Division Mécanique des Structures \\ Ecole Centrale de Nantes - 1, rue de La Noë \\ 44321 Nantes Cedex 3, France \\ laurent.gornet@ec-nantes.fr
}

\begin{abstract}
This paper deals with slamming phenomenon (impact between bow ship and water free surface). Slamming loads on ship may be sufficiently important so as to create plastic deformations of the hull external structure. In extreme cases, they have been recognised for being responsible for the loss of ships.

The problem to solve is transient and highly non-linear due to the character of the flow. In the present paper, the three-dimensional Wagner problem is solved numerically using a variational formulation together with a Finite Element Method. Threedimensional results for simple rigid bodies such as a cone and an ellipsoid are successfully compared with analytical results. Results for deformable structure will be presented.
\end{abstract}

\section{INTRODUCTION}

In rough seas where large relative amplitude ship motions occur, the bow may emerge out of the water. In these conditions of the state of the sea, the ship hull can experience impact loads as the bow reenters the incoming wave. This phenomenon is commonly referred as slamming. Despite the pressure field due to the impact remains localized in space and time, slamming loads can locally create plastic deformations to the hull external structure. In extreme cases, they have been recognized for being responsible for the loss of ships.

The purpose of this paper is to give some insight about this subject.
There are several approaches to study such a problem. On one hand, the Navier Stokes equations can be used to solve in velocity formulation, and on the other hand a velocity potential theory is introduced. In this paper, the second approach is going to be applied. The velocity potential equations are studied by an asymptotic analysis. The two dimensional problem has been widely studied and the theoretical analysis is now well established in case of a rigid body. First order analytical solutions have been found for a wedge with small deadrise angles (Wagner 1932), a cylinder (Cointe 1987), and arbitrary two dimensional blunt body shapes (Cointe 1989, Howinson, et al 1991). The latest consider an axisymetric body yet the results are closed to two-dimensional ones.

In above mentioned studies, the body is assumed to be rigid.

The two dimensional asymptotic solutions have been well compared to drop tests of different shapes (Cointe and Armand 1987, Fontaine and Cointe 1997, Zhao and al 1996, Magee and Fontaine 1998) and for rigid cone in three-dimensional (Faltinsen, 1997). For three-dimensional case, analytical solutions are far from possible expect in case of particular geometry (Scolan, Korobkin, 2001).

Korobkin (1995), Kvalsvold (1995), and Faltinsen (1997) studied ship bow behaviour modelling the structure as a beam so that the coupled fluid-structure mathematical problem can be solved simultaneously. It results that hydroelasticity have not to be neglected. More recently, Donguy, Peseux and Fontaine (2000) have 
shown that structural deformations present an important threedimensional character. So three-dimensional effects have to be taken into account.

This study takes place within this framework. We try to develop a purely numerical approach to solve general three dimensional problem of slamming, for rigid and deformable body, considering fully coupled fluid-structure interaction problem.

In a first part, the three dimensional governing equations for the fluid are presented. The setting equation relies on the asymptotic theory. For any geometry of body, the velocity potential formulation can not be solved analytically. So, a numerical resolution is performed by the finite element method. In a second part, the structure problem is considered and is classically solved by the finite element method. Then, we will focus on the coupled problem. The fluid structure interaction introduces a coupling matrix that we validate in two or three dimensional cases. Finally, we present solution of the coupled problem, which is iteratively solved by the Newmark time integration scheme.

\section{THREE-DIMENSIONAL PROBLEM 2.1 Exact formulation}

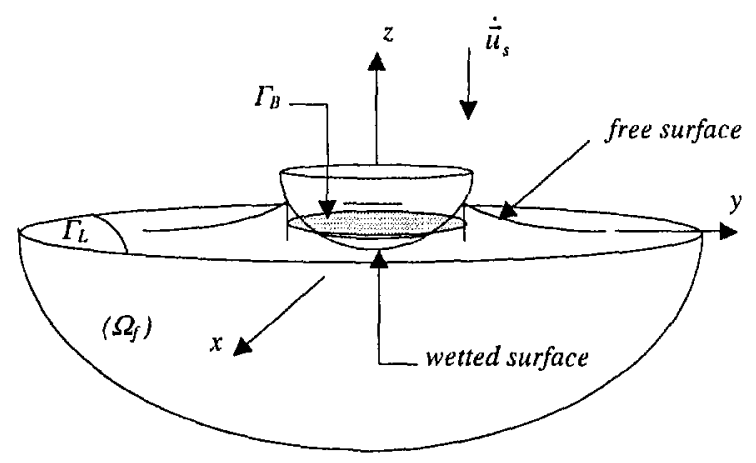

Figure 1. Geometrical definitions

We consider the problem of the impact of a three-dimensional body on the free surface (Fig. 1). The water entry problem is expressed within the framework of potential flow theory. Indeed, the fluid is assumed to be perfect and incompressible and the flow is irrotational. The velocity field can therefore be evaluated according to $\vec{v}=\operatorname{gra} d \phi$, where $\phi=\phi(x, y, z, t)$ is the velocity potential. Under these assumptions, the velocity potential must satisfy the following boundary value problem:

$$
\begin{array}{cl}
\Delta \phi=0 & \text { in } \Omega_{f} \\
\frac{\partial \phi}{\partial n}=\dot{\vec{u}}_{s} \cdot \vec{n} & \text { on the body } \\
\frac{\partial \phi}{\partial t}+\frac{I}{2}(g r \vec{a} d \phi)^{2}+\frac{p}{\rho}+g z=0 & \text { on the free surface }
\end{array}
$$

$$
\frac{\partial h}{\partial t}+\operatorname{gra} d \phi \cdot g r \vec{a} d h-\frac{\partial \phi}{\partial z}=0 \quad \text { on the free surface }
$$

where the displacement of the body is given by its $\vec{u}_{s}(x, y, t)$ and the free surface elevation is written as $z=h(x, y, t)$.

The body boundary condition, Eq. 2, expresses the continuity of the normal velocity component on the fluid-structure interface. The kinematic and dynamic free surface conditions, Eq. 3 and Eq. 4 respectively, state that the free surface is a material surface along which the pressure is constant. Moreover, the fluid is assumed to be initially at rest:

$$
h(x, y, 0)=0 \quad \phi(x, y, z, 0)=0
$$

and the flow to be unperturbed far from the body:

$$
|\operatorname{gra} d \phi| \rightarrow 0 \quad \text { when }\left(x^{2}+y^{2}+z^{2}\right)^{1 / 2} \rightarrow \infty
$$

Once the velocity potential is known, the pressure on the body is calculated by Bernoulli's equation:

$$
\frac{p}{\rho}=-\frac{\partial \phi}{\partial t}-\frac{1}{2}(g r \vec{a} d \phi)^{2}-g z
$$

\subsection{Asymptotic formulation}

The above mentioned boundaries value problem cannot easily be solved in $3 \mathrm{D}$, even numerically, due to the local and moving flow character. Classically, the hydrodynamic problem is simplified into:

$$
\begin{aligned}
\Delta \phi & =0 & & \text { in } \Omega_{f} \\
\frac{\partial \phi}{\partial n} & =\dot{\vec{u}}_{s} \cdot \vec{n} & & \text { on } \Gamma_{B} \\
\phi & =0 & & \text { on } \Gamma_{L} \\
\frac{\partial h}{\partial t} & =\frac{\partial \phi}{\partial z} & & \text { on } \Gamma_{L}
\end{aligned}
$$

where $\Gamma_{B}$ is the projection on $z=0$ of the wetted body surface and $\Gamma_{L}$ the position of the undisturbed free surface. $\Omega_{f}$ represent the linearized fluid domain (see Fig. 1). Cointe (1989), Wilson (1989) used the asymptotic expansion method to justify the mixte NeumanDirichlet problem. The perturbation procedure strongly relies on the blunt body assumption since the small parameter used in the asymptotic expansion is the ratio between the immersion parameter and the characteristic length scale of the body wetted width. Within a far field point of view, the body boundary condition, Eq. 2, can be written on $z=0$ without introducing significant error, therefore justifying Eq. 9. The exact dynamic condition, Eq. 3, has been replaced by a Dirichlet condition for the potential on the undisturbed position of the free surface, Eq. 10. Physically the acceleration in the fluid is assumed to be large compared to gravity which can be neglected during the first instants. Finally, the simplified kinematical 
free surface condition, Eq. 11, states that the vertical displacement of the free surface is equal to the fluid vertical motion, evaluated on the undisturbed position of the free surface. The quadratic terms have to remain small compared to the linear ones in order to keep valid this approximation. The resulting problem, Eqs. 8-11 is often referred to the generalised Wagner (1932) problem. In the present formulation, three-dimensional effects are retained through the Laplace's equation, and structural deformations of the body surface are taken into account in the body boundary condition.

\subsection{Numerical resolution}

The resolution procedure for the velocity and displacement potentials is based on the Finite Element Method. The weighted residual method is applied considering the weighting functions $\varphi$ which verifies the boundary condition $\varphi=0$ on $\Gamma_{L}$. The problem comes to minimize the integral quantity:

$$
W(\phi)=\int_{\Omega_{f}} \varphi R(\phi) d D
$$

where the residue $R(\phi)$ is set equal to $\Delta \phi$. Then, applying Green's identity and taking into account of boundary conditions, Eq. 13, leads to the weak formulation:

$$
\int_{\Omega_{J}} \operatorname{gra} d \varphi \cdot g r \vec{a} d \phi \cdot d D=\int_{\Gamma_{s}} \varphi\left(\dot{\vec{u}}_{s}, \vec{n}\right) \cdot d S
$$

The potential $\phi$ and weight functions $\varphi$ are approximated using Galerkin's method leading to:

$$
\varphi=\left\langle N_{f}\right\rangle\langle\varphi\}^{e} \quad \phi=\left\langle N_{f}\right\rangle\langle\phi\}^{e}
$$

where the shape functions $N_{f}$ depend on the types of elements, and $\langle\phi\rangle^{e}$ denotes the nodal potential vector of the finite element $(e)$. Finally, the following linear system is deduced from the discretization of the bilinear forms, Eq. 13:

$$
\begin{gathered}
{[H]\{\phi\}=\left\{G_{\phi}\right\}} \\
H_{i j}^{e}=\int_{\Omega^{\cdot}} N_{i, j} N_{j, i} d D \\
\left\{G_{\phi}\right\}^{e}=\int_{I_{B}^{e}}\left\{N_{f} \gamma\left(\dot{\vec{u}}_{s} \cdot \vec{n}\right) d S\right.
\end{gathered}
$$

To evaluate the right hand side of eq. (17), it is necessary to know the wetted portion of the body $\Gamma_{B}$ or, equivalently, the position of the contact line between the body and the fluid. Due to the deformations of the free surface, the wetted surface is part of the unknown of the problem. An additional equation is therefore needed to close the problem. Physically, the contact line is determined by imposing to the solution to satisfy volume of the fluid conservation (Wilson, 1989, Fontaine \& Cointe, 1992) or equivalently, by imposing the existence of an intersection point between the elevation of the free surface and the body as intuitively done by Wagner (1932). Volume conservation should be automatically satisfied since Laplace's equation is solved, the wetted surface beeing determined through an iterative procedure. (Donguy, Peseux and Fontaine, 2000)

Once convergence has been reached, the position of the contact line is known. The linear system for the velocity potential is then solved. As a result, a simple first order backward finite difference scheme gives an accurate estimation of the pressure. This last solution represents only the fluid flow far from the body. Consequently, pressure distribution is singular close to the boundary wetted surface. This singularity is corrected by matching numerical solution with the analytical solution of the flow near the body.

\section{HYDROELASTICITY}

In this section, the previous analysis is extended to treat the case of deformable body. The equations governing the structural deformations are first recalled. A coupled method to solve the fluidstructure interaction problem is then proposed.

\section{$\underline{3.1}$ Governing structural equations}

The usual assumptions of small perturbations is made. The equilibrium equation reads:

$$
\rho \ddot{\vec{u}}_{s}=d \vec{i} v_{d} \overline{\bar{\Sigma}}+\vec{f} \quad \text { in } \Omega_{s}
$$

where $\overline{\bar{\Sigma}}$ denotes the stress tensor, $\vec{u}_{s}$ corresponds to the structural displacement, and $f$ corresponds to volume force associated for example with the gravity field. If gravity effects do not contribute to the local deformations here, they nevertheless influence the global motion of the body before impact. Boundary condition on the structure is:

$$
\overline{\bar{\Sigma}}_{f} \cdot \vec{n}=\overline{\bar{\Sigma}}_{s} \cdot \vec{n} \quad \text { on } \Gamma_{B}
$$

where the subscript $f$ and $s$ refer to the fluid and the structure, respectively,

The structural modeling is performed within the framework of linear elasticity. Hooke's law is used to express the relation between the stresses and the strains which are given by the linearised GreenLagrange tensor $\varepsilon$ :

$$
\varepsilon_{i, j}=\frac{I}{2}\left(u_{i, j}+u_{j, i}\right)
$$

\subsection{Coupled problem}

The structural problem is also solved using the Finite Element Method. The principle of virtual works is applied to Eq. 18 together with boundary condition, Eq. 19 , leading classically to the discretised form:

$$
\left[M_{s}\right]\{\ddot{U}\}+[K]\{U\}=\{F\}
$$




$$
\{F\}^{e}=\int_{\Gamma_{B}^{e}}\left[N_{s} \Gamma\{n\} p d S\right.
$$

where the mass and stiffness matrices, respectively $\left[M_{s}\right]$ and $[K]$, together with the generalized load vector $\{F\}$, are obtained by assembly of the elementary terms calculated on each finite element $(e)$. The nodal displacements $(U)$ is linked to the interpolation of the displacement field through:

$$
\left\{u_{s}\right\}=\left[N_{s}\right]\{U\}_{e}
$$

By definition, hydroelastic effects arise as a result of a strong interaction between the fluid flow and the structural response. From Eq. 22, it can be seen that the pressure imposed by the fluid will drive the structural deformations. The structural deformations influence the pressure field through the body boundary condition as shown by Eq. 17. In order to obtain a robust method to solve the coupled fluid structure interaction problem, all the evolution equations are integrated simultaneously. The structural deformations are included in the fluid flow discrete formulation, while the pressure is expressed as the time derivative of the velocity potential in the discrete structural analysis. The following problem is then obtained:

$$
\begin{aligned}
& {[H]\{\phi\}=[F S]\{\dot{U}\}} \\
& \left.\left[M_{s}\right\} \ddot{U}\right\}+[K]\{U\}=-\rho_{f}[F S\}\{\dot{\phi}\} \\
& {[F S]^{e}=\int_{\Gamma_{B}^{e}}\left\{N_{f}\right)\{n\}\left[N_{s}\right\} d S}
\end{aligned}
$$

where the coupling terms $[F S]$ representing the fluid - structure interaction appear explicitly. Eq. 26 shows that the coupling term involves only the values of the structural displacement $\vec{u}_{s}$ and the velocity potential $\phi$ on the wetted surface of the body, $\Gamma_{B}$.

\subsection{Validation of the coupling matrix in 2D}

The numerical resolution of Eq. 24 or Eq. 25 is performed using commercial Finite Element Method numerical code Castem. An external procedure is implemented for the evaluation of the coupling matrix $[F S]$. The correct implementation of the approach is checked for the test case problem of sloshing in a rectangular tank including an elastic beam in its center, as shown in Figure 2 for twodimensional case. This problem has been studied experimentally by Chaï (Chaï, 1996).

For this problem, gravity cannot be neglected and so the formulation introduces a mass matrix. We obtained the following problem (Morand and Ohayon, 1992):

$$
\begin{aligned}
& {\left[M_{f}\right]\{\dot{\phi}\}+[H]\{\phi\}=[F S]\{\dot{U}\}} \\
& {\left[M_{s}\right]\{\ddot{U}\}+[K]\{U\}=-\rho_{f}[F S]\{\dot{\phi}\}} \\
& {[F S]^{e}=\int_{I_{B}^{e}}\left(N_{f} R\{n\}\left[N_{s}\right] d S\right.}
\end{aligned}
$$

The mechanical excitation consists in a harmonic load applied to the beam or the plate extremity. A frequency scanning is performed, see Fig. 4, to evaluate the eigenfrequencies which correspond to the peaks of the beam displacement. The free surface elevation for the first four modes of the coupled problem is shown in Fig. 3, together with the modal shape of the elastic beam. The numerical values of the eigenfrequencies are presented in table 1 , column $\mathrm{Cl}$. They are compared to the results by Peseux et al (1999), column entitled "Ref". A toolbox that allows the direct computation of the resonant frequencies was also applied, see column $\mathrm{C} 2$. All the results are relatively close to each other, especially, the two last columns. The computation by Peseux was performed using another f.e.m. code, which may explains the small differences in the results. The agreement between the three methods is very satisfactory.

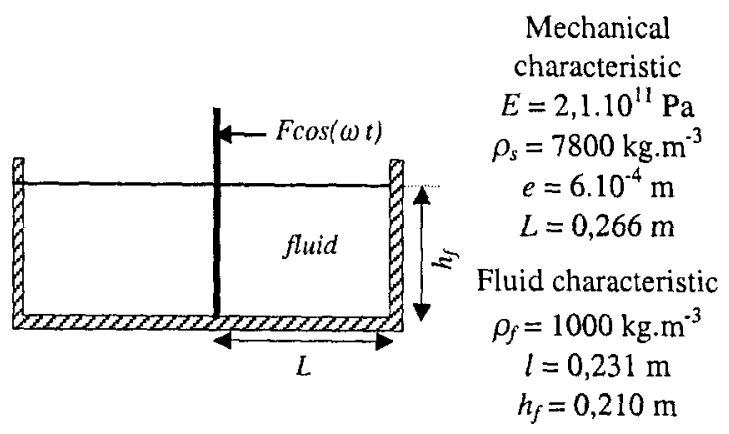

Figure 2. Elastic beam in a heavy fluid problem

\begin{tabular}{|c|c|c|c|}
\hline Hertz & Ref. & $\mathrm{C} 1$ & $\mathrm{C} 2$ \\
\hline $\mathrm{f}_{1}$ & 1.49 & 1.60 & 1.61 \\
\hline $\mathrm{f}_{2}$ & 1.86 & 1.84 & 1.83 \\
\hline $\mathrm{f}_{3}$ & 2.39 & 2.46 & 2.44 \\
\hline $\mathrm{f}_{4}$ & 2.66 & 2.63 & 2.61 \\
\hline $\mathrm{f}_{5}$ & 2.97 & 3.06 & 3.01 \\
\hline $\mathrm{f}_{6}$ & 3.33 & 3.29 & 3.21 \\
\hline $\mathrm{f}_{7}$ & 3.46 & 3.51 & 3.42 \\
\hline
\end{tabular}

Table 1. Eigenfrequencies in $\mathbf{H z}$

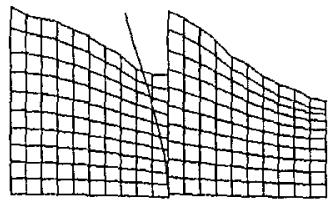

$\mathrm{f}=1,6 \mathrm{~Hz}$

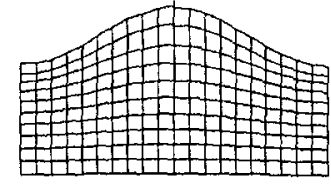

$\mathrm{f}=1,84 \mathrm{~Hz}$ 


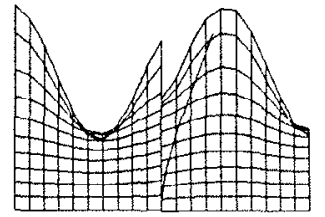

$\mathrm{f}=2,46 \mathrm{~Hz}$

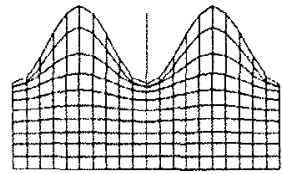

$\mathrm{f}=2,63 \mathrm{~Hz}$
Figure 3. Eigenmodes shapes

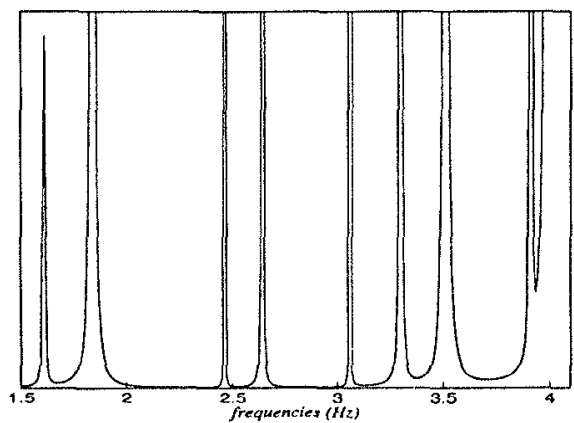

Figure 4. Eigenfrequencies search using scanning

\subsection{Validation of the coupling matrix $\ln 3 \mathrm{D}$}

To validate the coupling matrix in three-dimensional case, we are interested in the test case problem of sloshing in a rectangular tank including an elastic plate in its center, as shown in Figure 5a. The figure $5 \mathrm{~b}$ shows the finite elements mesh used, which are hexaedron elements for the fluid and quadrilateral elements for the structure. The equations of the problem are the same as the Eq. 27 - Eq. 29.

Once again, the mechanical excitation consists in a harmonic load applied to the plate extremity. A frequency scanning is performed, see Fig. 6, to evaluate the eigenfrequencies which correspond to the peaks of the plate displacement. The free surface elevation with the modal shape of the plate beam for the first four modes of the coupled problem is shown in Fig. 7. In table 2, we reported the numerical values of the eigenfrequencies are presented in table 2. Morever, experimental results for plate vibration (Peseux, 1989) are given in the column "Exp". Comparison of frequencies leads to good agreement, which give confidence into the extemal numerical procedure that has been developed in the present study. Developing this procedure, and controlling precisely the coupling terms resulting from the fluid - structure interaction is necessary to treat the water impact problem of an elastic body.

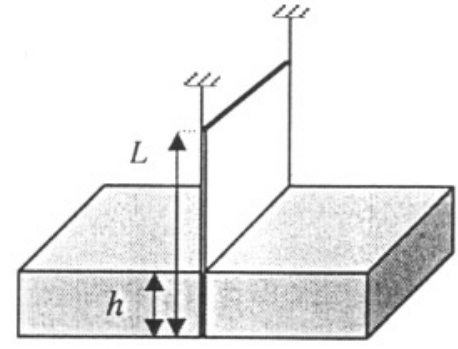

Mechanical

characteristic

$E=2,1.10^{11} \mathrm{~Pa}$

$\rho_{s}=7800 \mathrm{~kg} \cdot \mathrm{m}^{-3}$

$e=5.7 .10^{-3} \mathrm{~m}$

$L=0,40 \mathrm{~m}$

Fluid characteristic

$\rho_{f}=1000 \mathrm{~kg} \cdot \mathrm{m}^{-3}$

$h=0,10 \mathrm{~m}$

Figure 5a. Elastic plate in a heavy fluid problem

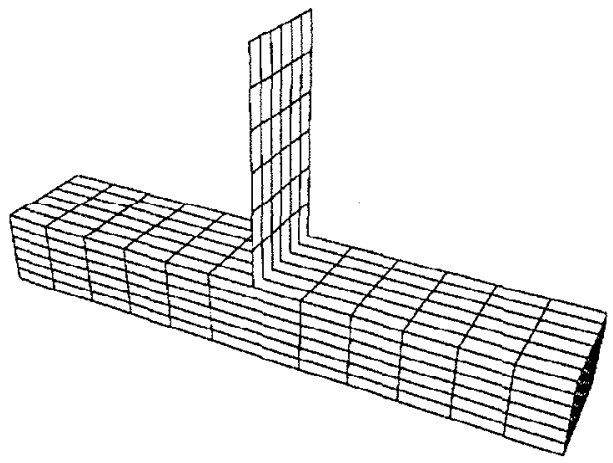

Figure $5 b$. Mesh of the elastic plate problem

\begin{tabular}{|c|c|c|c|c|}
\hline Hertz & Ref. & $C 1$ & $C 2$ & Exp. \\
\hline$f_{1}$ & 171.1 & 171.5 & 170.1 & 170.9 \\
\hline$f_{2}$ & 266,4 & 260,5 & 258.9 & 265.3 \\
\hline$f_{3}$ & 500,1 & 543,5 & 525.6 & 484.4 \\
\hline$f_{4}$ & 610,5 & 626,5 & 617.9 & 597.5 \\
\hline$f_{6}$ & 1037 & 1114 & 1083 & 941 \\
\hline$f_{7}$ & 1052 & 1152 & 1089 & 998 \\
\hline$f_{8}$ & 1341 & 1316 & 1265 & 1228 \\
\hline
\end{tabular}

Table 2. Eigenfrequencies in $\mathbf{H z}$

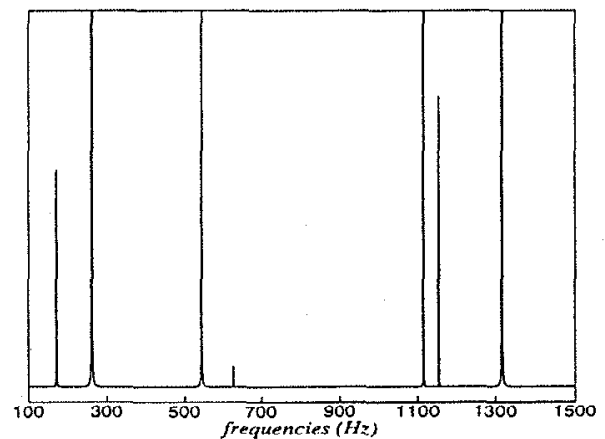

Figure 6. Elastic plate in a heavy fluid problem 

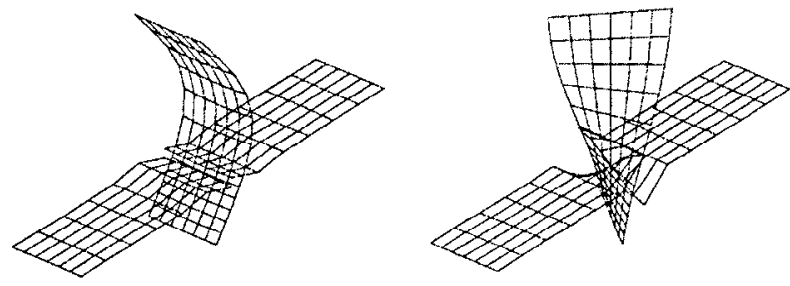

$\mathrm{f}=171,1 \mathrm{~Hz}$

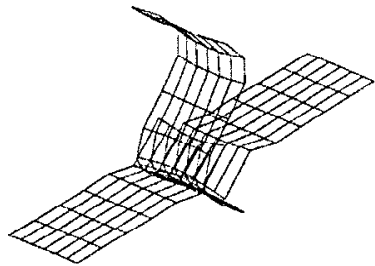

$\mathrm{f}=500,1 \mathrm{~Hz}$

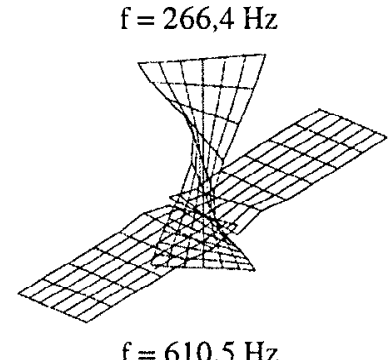

$\mathrm{f}=610,5 \mathrm{~Hz}$

Figure 7. Eigenmodes shapes

\subsection{Numerical simulation of the coupled problem}

One way for the coupled numerical resolution is to rewrite Eqs. 27 and 28 using with a ncw nodal vector $\{W\}^{T}=\{\phi, U\}^{T}$, leading to:

$$
[M]\{\ddot{W}\}+[B]\{\dot{W}\}+[K]\{W\}=0
$$

where:

$$
[M]=\left[\begin{array}{cc}
{[0]} & {[0]} \\
{[0]} & {\left[M_{f}\right]}
\end{array}\right] \quad[B]=\left[\begin{array}{cc}
{[0]} & -[F S] \\
\rho_{f}[F S\} & {[0]}
\end{array}\right] \quad[\mathrm{K}]=\left[\begin{array}{cc}
{[H]} & {[0]} \\
{[0]} & {\left[K_{s}\right]}
\end{array}\right]
$$

The fluid - structure interaction problem, Eq. 31 , is then integrated starting from the initial conditions:

$$
\begin{aligned}
& W(t=0)=W_{o} \\
& \dot{W}(t=0)=\dot{W}_{o}
\end{aligned}
$$

in a numerical temporal explicit or implicit scheme with a time step $d t$. Nevertheless, studying for rigid body slamming, it appears that time step $d t$ for the fluid problem has to be less than the fiftieth part of the instant to calculate accurately pressure distribution. Choosing the same time step for the coupled problem simulation leads to generate a high costly time procedure.

Consequently, we solved the fluid-structure interaction problem with an iterative procedure. The fluid problem is first solved with a step time $\delta t$. After determination of the force due to the pressure, the structural resolution is within the Newmark temporal integrated sheme with the step time $\Delta t$. The time schema is represented by Figure 8 :

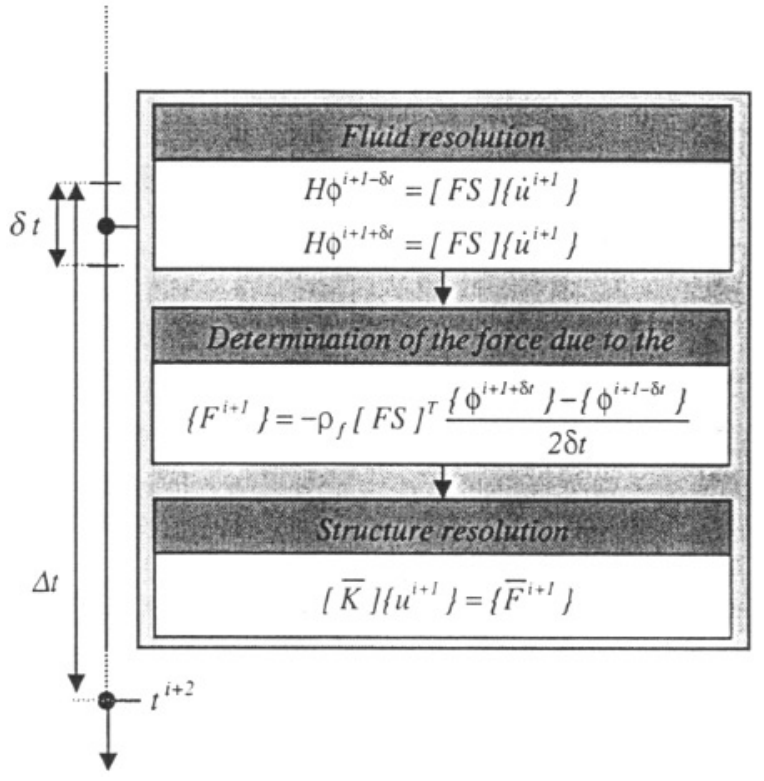

Figure 8. Iterative scheme used to solved the coupled problem

Experimental drop tests of elastic cones have been realized at the Mechanic and Material Laboratory of the Ecole Centrale of Nantes (Donguy, 2002). The numerical results have been compared to the experimental ones obtained for the impact of cone with thickness $1.5 \mathrm{~mm}$ and deadrise angle $14^{\circ}$. The figure 9 represent the pressure history at two points of the structure. The structural deformation present a strongly axisymetric character as shown in the figure 10 .

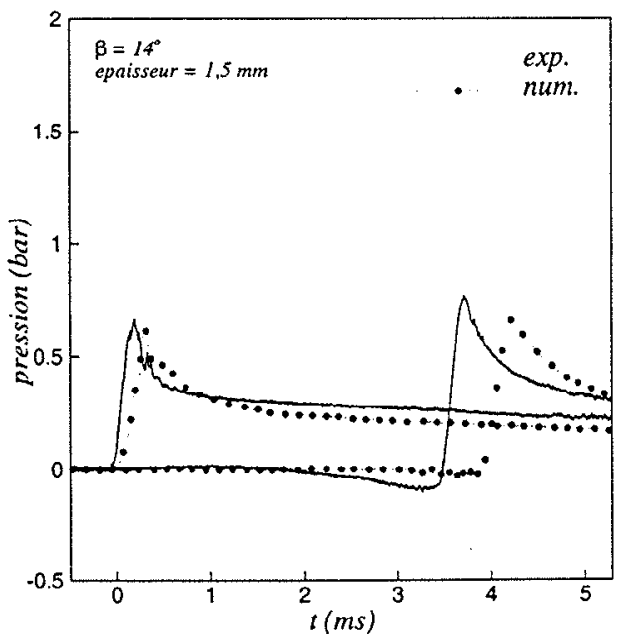

Figure 9. Comparison of pressure history for the impact of a cone with thickness $1,5 \mathrm{~mm}$ (deadrise angle $14^{\circ}$ ). In dot line : numerical solution - In straight line : experimental values 


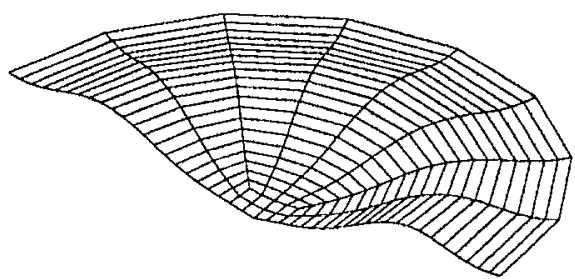

Figure 10. Shape deformation of the structure with the coupled resolution

\section{CONCLUSION}

In the present paper, a numerical method is proposed to solve the three-dimensional hydrodynamic impact problem for blunt bodies.

In a first part, we have presented the fluid problem formulated in velocity potential and relying on asymptotic theory. The equations deduced from such formulation have been solved through the FEM. In a second part, we have examined the structural governing equations, which have been classically resolved by FEM as well. Finally the two previous methods had been used to treat the case of deformable bodies. Yet this formulation introduces a coupling matrix. The correct evaluation of the terms has been checked for the sloshing problem of a tank with an elastic beam in two-dimensional case and elastic plate in three-dimensional case. The coupling problem has been integrated in time by a Newmark schema with an iterative coupling. The results that we have obtained under these assumptions are rather good.

This formulation entails the boundary conditions projection. To avoid this assumption, and follow the evolution of the free surface, other method can be used like the ALE (Arbitrary Lagrangian Eulerian) method.

\section{REFERENCES}

Chai, X.J. (1996) "Influence de la gravité sur les interactions fluidestructure pour un fluide dans un domaine borné à surface libre", Thesis, Institut National Polytechnique de Lorraine.

Cointe, R. (1987) "L'impact hydrodynamique en deux dimensions", $I^{\text {ère }}$ Journées de l'Hydrodynamique, Nantes.

Cointe, R., Armand, J.L. (1987) "Hydrodynamic impact analysis of a cylinder", J. Offshore Mechanics and Artic Engineering, vol 9, pp 237-243.

Cointe, R. (1989) "Two dimensional water solid impact", Journal of Offshore Mechanics and Arctic Engineering, vol 111, pp 109-114.

Donguy, B., Peseux., B, Fontaine, E. (2000) "On the ship structural response due to slamming loads", Proc. of European Congress on Computational Methods in Applied Sciences and Engineering, Barcelona.

Donguy, B. (2002) "Etude de l'intéraction fluide-structure lors de l'impact hydrodynamique", Thèse, Ecole Centrale de Nantes.

Faltinsen, O., Zhao, R. (1997) "Water entry of ship sections and axisymmetric bodies", High Speed Body Motion in Water.
Faltinsen, O. (1997) "The effects of hydroelasticity on ship slamming", Phil. Trans. R. Soc. Lond., vol. 355, pp. 575-591.

Fontaine, E., Cointe, R. (1992) "A second-order solution for the wedge entry with small deadrise angle", $7^{\text {th }}$ International Workshop on Water Waves and Floating Bodies, Val de Reuil.

Fontaine, E., Cointe, R. (1997) "Asymptotic theory of water entry", High Speed Body Motion in Water, Kiev, NATO conference.

Howison, S.D., Ockendon, J.R., Wilson S.K. (1991) "Incompressible water entry problems at small deadrise angles", Journal of Fluid Mechanics, vol 222, pp 215-230.

Korobkin, A.A. (1995) "Wave impact on the bow end of a catamaran wetdeck", Journal of Ship Research, vol 39, pp 321-327.

Kvalsvold, J., Faltinsen, O.M. (1995) "Hydroelastic modeling of wet deck slaming on multihull vessels", Journal of ship Research, vol 39, pp 225-239.

Magee, A., Fontaine, E. (1998) "A coupled approach for the evaluation of slamming loads on ships", Proc. $7^{\text {th }}$ Int. Symp. on Practical Design of Ships and Mobil Units, The Hague Netherlands.

Morand, H.J.-P., Ohayon, R. (1992) "Interactions FluidesStructures", Recherche en Mathématiques Appliquées, Masson editor.

Peseux, B. (1989) "Contribution à l'étude de structure partiellement immergées en matériau homogène ou en composite", Ecole Nationale Supérieure de Mécanique de Nantes.

Peseux, B., Cartraud, P., Argouar'ch, Y. (1999) "Dynamic response of partially filled tanks - Explicite time integration method", Proc. of ASME Pressure Vessels and Pipe Conference, vol 394, pp 191. 199.

Scolan, Y.M., Korobkin, A.A. (2001) "Three-dimensional theory of water impact. Part 1. Inverse Wagner problem", Journal of Fluid Mechanics, vol 440, pp 293-326.

Wagner, H. (1932) "Über Stoss und Gleitvorgänge an der Oberfläche von Flüssigkeiten", Z. Ang. Math. Mech., vol 12, pp 193-215.

Wilson, S.K. (1989) "The mathematics of ship slamming", Ph.D. thesis, University of Oxford.

Zhao, R., Faltinsen, O.M., Aarsnes, J.V. (1996) "Water entry of arbitrary two-dimensional sections with and without flow separation", Proc. $21^{\text {st }}$ Symposium on Naval Hydrodynamic, Trondheim, Norway. 